\title{
Long-Term Benefit and Withdrawal Effect of Statins After Percutaneous Coronary Intervention: A Nationwide Population-Based Cohort Study
}

This article was published in the following Dove Press journal: Patient Preference and Adherence

\author{
Hye Yun Jeong' \\ So-Young Lee' \\ Sang Hoon $\operatorname{Kim}\left(\mathbb{D}^{2, *}\right.$ \\ Jinkwon $\operatorname{Kim} \mathbb{1}^{3, *}$ \\ 'Division of Nephrology, Department of \\ Internal Medicine, CHA Bundang Medical \\ Center, CHA University School of \\ Medicine, Seongnam, Korea; ${ }^{2}$ Division of \\ Cardiology, Department of Internal \\ Medicine CHA Bundang Medical Center, \\ CHA University School of Medicine, \\ Seongnam, Korea; ${ }^{3}$ Department of \\ Neurology, Yongin Severance Hospital, \\ Yonsei University College of Medicine, \\ Yongin-si, Korea
}

*These authors contributed equally to this work
Correspondence: Jinkwon Kim Department of Neurology, Yongin Severance Hospital, Yonsei University

College of Medicine, 363,

Dongbaekjukjeon-daero, Giheung-gu,

Yongin-si 16995, Korea

Tel +82-3I-5I89-8I52

Fax +82-3I-5I89-8208

Email antithrombus@gmail.com

Sang Hoon Kim

Division of Cardiology, Department of Internal Medicine, CHA University School of Medicine, CHA Bundang Medical

Center, 59 Yatap-ro, Bundang-gu,

Seongnam-si 13496, Korea

Tel +82-3I-780-5585

Fax +82-3I-780-5857

Email kimsang978@naver.com
Purpose: Despite indubitable evidence for the cardiovascular benefits of statins, there have been concerns that statin discontinuation may cause negative effects known as "statin withdrawal syndrome." This study aimed to assess the benefit and the withdrawal effect of statins after percutaneous coronary intervention (PCI).

Patients and Methods: We conducted a retrospective cohort study on 5218 patients who underwent PCI between 2002 and 2013 using the nationwide health insurance claim data in Korea. Based on the prescription data, the use of statins during follow-up was classified into three risk periods: "statin period" (period with statin cover), "statin withdrawal period" (withdrawal of statin within 30 days), and "no statin period" (no exposure to statin for longer than 30 days). The primary outcome was the composite outcome of myocardial infarction, coronary revascularization, stroke, and all-cause death. We performed multivariate Cox proportional regression analyses which treated the use of statins as a time-dependent variable.

Results: During the follow-up period of $3.54 \pm 2.91$ years (mean \pm standard deviation), 1515 (29.0\%) patients sustained a primary outcome. Compared with the "no statin period," the "statin period" was associated with lower risk of the primary outcome (adjusted hazard ratio [HR] 0.72 , 95\% confidence interval [CI, 0.63-0.81]). While the "statin withdrawal period" posed a significantly increased risk (adjusted HR 1.87, 95\% CI [1.52-2.29]). With respect to the intensity of statins associated with withdrawal, dose-dependent increased risk was observed for withdrawal of low-, moderate-, and high-intensity statins; adjusted HR [95\% $\mathrm{CI}$ ] were 1.45 [0.74-2.86], 1.86 [1.49-2.32], and 2.61 [1.41-4.81], respectively.

Conclusion: After PCI, there was an increased cardiovascular risk during the statin withdrawal period, especially with the use of high-intensity statins. To maximize the beneficial effect and to avoid the withdrawal effect of statins, high-risk patients need to adhere to taking statins without discontinuation.

Keywords: statin, percutaneous coronary intervention, statin withdrawal, adherence

\section{Introduction}

Coronary artery disease (CAD) is the leading cause of morbidity and mortality worldwide. Statins (3-hydroxy-3-methylglutaryl-CoA reductase inhibitor) are a class of lipidlowering agents, which have been established to be effective in reducing cardiovascular risk. $^{1-3}$ Current guidelines strongly recommend lifelong statin therapy for high-risk patients with established CAD. ${ }^{4,5}$ Despite the established cardiovascular benefits, statin use is dynamic or intermittent in clinical practice, and suboptimal statin use is frequent. ${ }^{6-8}$ There is cumulative evidence that discontinuation and non-adherence to the use of statins 
are strongly associated with poor cardiovascular prognosis. ${ }^{9-11}$ However, knowledge of statin use is still limited throughout the long-term follow-up period in patients with CAD. Furthermore, sudden discontinuation of statins may cause additional adverse effects (statin rebound or withdrawal syndrome). ${ }^{12-14}$ To evaluate the benefit and the withdrawal effect of statins in a long-term follow-up period, we conducted a retrospective cohort study on patients who underwent percutaneous coronary intervention (PCI) using the nationwide health insurance claim data.

\section{Materials and Methods Data Source}

In this study, we used the data from the National Health Insurance Service-National Sample Cohort (NHIS-NSC) in Korea. ${ }^{15}$ South Korea has universal health insurance coverage by the NHIS under their national plans. The NHIS-NSC included 1,025,340 individuals selected in 2002 (2.2\% of the total eligible Korean population) by stratified random sampling according to sex, age, and household income. The NHIS-NSC contains information on all health claim data between 2002 and 2013 including hospital visits, medical procedures, drug prescriptions, diagnoses, and mortality (cause and date of death). The diagnosis at each hospital visit was recorded according to the International Classification of Diseases, 10th revision (ICD-10).

\section{Study Design}

This was a retrospective cohort study based on the NHISNSC. We included subjects who were aged $\geq 20$ years and who underwent PCI (angioplasty with or without stent; they had health claim codes of "M6561", "M6562”, "M6563", "M6564", "M6551", and "M6552”) in 2002-2013. To minimize confounding bias by cancer, we excluded patients who had a diagnosis code of malignant neoplasms (C00-C97) during the study period. Index date for each patient was defined as the admission date for PCI. The primary outcome was a composite of myocardial infarction (MI), coronary revascularization, stroke (ischemic and hemorrhagic), and all-cause mortality, whichever occurred first after the index date. MI was defined as hospitalization (admission or emergency department visit) with a primary diagnosis of I21. Coronary revascularization was determined by health claim codes of PCI ("M6561," "M6562," “M6563," "M6564," "M6551," "M6552”) and coronary artery bypass graft (“OA631*_OA639*”, “OB631*_OB639*”, “OA641*”,
“OA642*”, “OA647*”, “O0161*_O0171*”, “O1641*_ O1647*". ${ }^{16}$ If a patient admitted with MI and received coronary revascularization during the hospitalization period, the case was classified as MI. Stroke was diagnosed in patients hospitalized with a primary diagnosis of I60-63 (ischemic stroke, I63; hemorrhagic stroke, I60-62) and who underwent brain CT or MRI during the hospital visit. In previous validation studies with the NHIS, the diagnostic accuracy of I21 for MI was reported as 73-93\%, and I60-I63 for stroke as over $80 \% .{ }^{17-20}$ Several research articles have been published based on the NHIS health claim data on MI and stroke. ${ }^{21-23}$ Unless the patient was disqualified from the NHIS because of death or emigration, all included patients were followed until December 2013 or the date of the primary outcome. As a very short period of follow-up was inadequate to evaluate long-term prognosis following the use and the withdrawal of statins, those followed up for $\leq 30$ days were excluded. All data in the NHIS-NSC were fully anonymized. Therefore, this study was approved, and informed consent was waived, by the Institutional Review Board of Bundang CHA Medical Center (CHAMC 2017-03-021).

\section{Prescription Data Including Statin}

All prescription data of included patients are available in the NHIS-NSC. According to the prescription data of statins, statin treatment during follow-up was classified into three risk periods: "statin period" (days covered by statins), "statin withdrawal period" (within 30 days from the end of the most recent statin exposure), and "no statin period" (no statin exposure for more than 30 days). Figure 1 illustrates examples of the risk periods according to the respective statin treatment regimes. In the "statin withdrawal period," the intensity of the last used statins (low, intermediate, or high) was determined by the daily dose and type (atorvastatin, fluvastatin, rosuvastatin, pitavastatin, pravastatin, simvastatin, and lovastatin) according to the classification in the 2013 American Heart Association guideline on the treatment of high blood cholesterol. ${ }^{4}$ As a covariate, we also collected data on the use of aspirin and adenosine diphosphate (ADP) receptor antagonist (clopidogrel, ticlopidine, ticagrelor and prasugrel). The use of statins and antiplatelets during follow-up was treated as a time-dependent variable for analyses.

\section{Other Covariates}

We identified the data on sex, age (classified into five-year groups in the NHIS-NSC), and household income as 


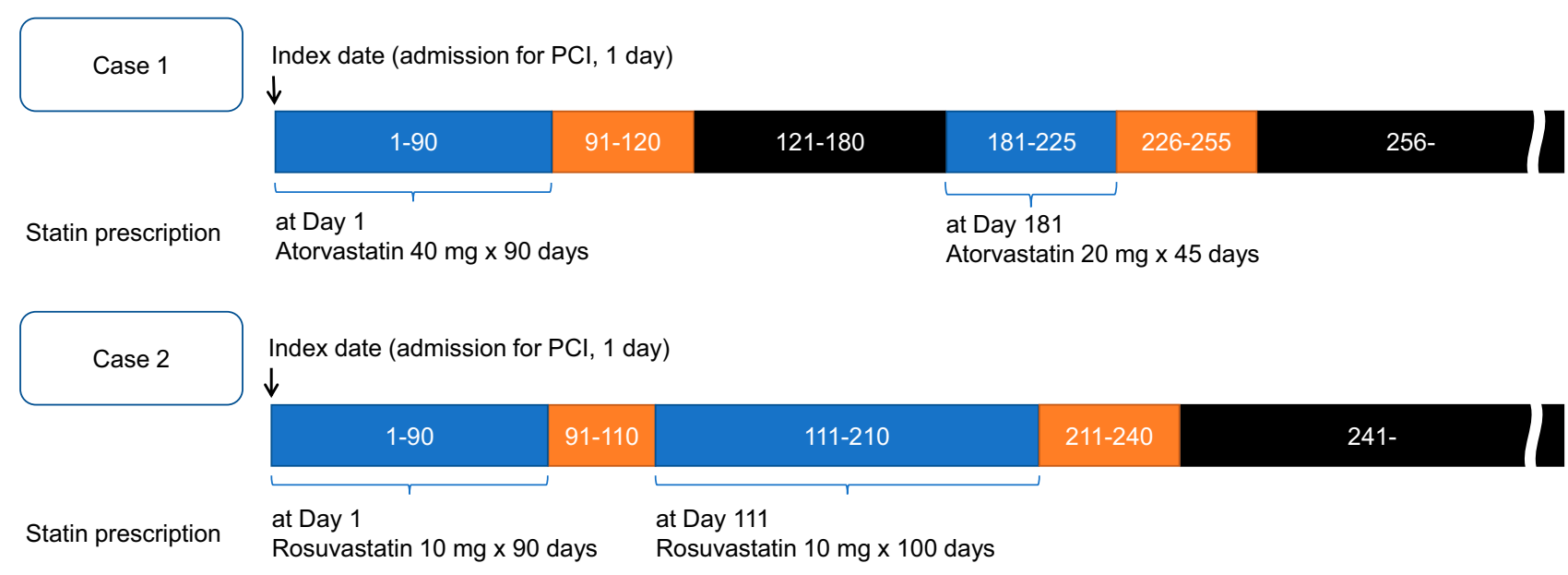

Figure I Examples of the definition of risk periods according to statin treatment. Abbreviation: $\mathrm{PCl}$, percutaneous coronary intervention.

a marker of socioeconomic status in the year in which PCI was undertaken. Household income obtained from the NHIS-NSC was subdivided into tertile groups (low, middle, and high) for analytical convenience. Hypertension (I10-15), diabetes mellitus (E08-11, E13-14), and atrial fibrillation (I48) were defined based on the presence of the diagnostic codes (ICD-10) in the NHIS-NSC before or at the time of discharge after PCI. Hypertension and diabetes mellitus were recognized as relevant only if the subjects received antihypertensive (calcium-channel blockers, angiotensin-converting enzyme inhibitors, angiotensinreceptor blockers, diuretics, beta-blockers, alphablockers, or vasodilators) or antidiabetic (sulfonylureas, biguanides, alpha-glucosidase inhibitors, thiazolidinediones, meglitinides, glucagon-like peptide-1 receptor agonists, dipeptidyl peptidase- 4 inhibitors, or insulin) agents in combination with the respective diagnostic codes. ${ }^{24}$ A history of MI was determined when a patient was admitted with a diagnosis of "I 21 " before or upon the decision to perform PCI.

\section{Statistical Analysis}

We computed the hazard ratio (HR) and 95\% confidence interval (CI) for the primary outcome using timedependent Cox proportional hazard regression analyses. The assumption of proportional hazards in the Cox models was tested by evaluating scaled Schoenfeld residuals, which were found to be satisfactory. Adjustments were performed for sex; age (as continuous variables); level of household income; presence of hypertension, diabetes mellitus, atrial fibrillation, and MI (as time-independent variables); and use of antiplatelets, and statins during follow-up (as time-dependent variables). As secondary outcome analysis, we constructed individual Cox regression models for each clinical outcome (MI, coronary revascularization, stroke, and all-cause mortality). The data manipulation and statistical analyses were performed with PostgreSQL version 10.1 (The PostgreSQL Global Development Group; https://www.postgresql.org/) and $\mathrm{R}$ software version 3.4.1 (The $\mathrm{R}$ Foundation for Statistical Computing, Vienna, Austria; http://www. R-project.org/). A two-sided $P$-value of $<0.05$ was considered statistically significant.

\section{Data Availability}

Requests for access to the NHIS data can be made through the homepage of National Health Insurance Sharing Service [http://nhiss.nhis.or.kr/bd/ab/bdaba021eng.do]. To gain access to the data, a completed application form, a research proposal, and the applicant's approval document from the institutional review board should be submitted to and reviewed by the inquiry committee of research support in the NHIS. The NHIS-NSC data were fully anonymized and did not contain any information that allowed patient identification.

\section{Results}

In the NHIS-NSC, there were 7682 adult patients (age $\geq 20$ years) who underwent PCI between 2002 and 2013. After 
Table I Clinical Characteristics of Patients Who Received Percutaneous Coronary Intervention

\begin{tabular}{|l|l|}
\hline Variable & [AII] N = 52 I 8 \\
\hline Sex, male & $3402(65.2)$ \\
Age & $60-64[50-54 ; 70-74]$ \\
Hypertension & 45 II (86.5) \\
Diabetes mellitus & $1742(33.4)$ \\
Atrial fibrillation & $436(8.4)$ \\
Myocardial infarction & $2380(45.6)$ \\
\hline Household Income & \\
Low & $1535(29.4)$ \\
Middle & $1908(36.6)$ \\
High & $1775(34.0)$ \\
\hline Year of Admission & \\
$2002-2005$ & $1059(20.3)$ \\
$2006-2009$ & $1805(34.6)$ \\
$2010-2013$ & $2354(45.1)$ \\
\hline
\end{tabular}

Note: Data are number (\%) or median [interquartile range].

excluding 2464 patients who were followed up for $\leq 30$ days or who had diagnosis of malignant neoplasms, we finally included 5218 patients. Among the 5218 included patients, $65.2 \%$ were male, and the median age was $60-64$ years (Table 1). The total study follow-up period of study patients was 18,049 person-years. Of them, the proportion of days-covered by any statins were $71.3 \%(12,866$ person-years). In terms of the percentage of individual statins used, atorvastatin was the highest $(44.9 \%)$, and rosuvastatin $(23.0 \%)$ and simvastatin $(16.1 \%)$ were second and third. During the follow-up period of $3.54 \pm 2.91$ years (mean \pm standard deviation), there were 1515 patients (29.0\%) who suffered primary outcome events (358 with MI, 670 with coronary revascularization, 181 with stroke, and 303 with all-cause mortality; considering only the earliest outcome for each patient).

In a time-dependent Cox regression analysis (Table 2), the "statin period" was significantly associated with lower risk of the primary outcome after PCI compared with the "no statin period" (adjusted HR [95\% CI], 0.72 [0.63-0.81], p < 0.001). While the "statin withdrawal period" resulted in a significantly increased risk of the primary outcome (adjusted HR [95\% CI], 1.87 [1.50-2.29], $\mathrm{p}<0.001)$. Further analysis with respect to the intensity of the statin used just before withdrawal revealed

Table 2 Result of Cox Regression Analyses for Primary Outcome After Percutaneous Coronary Intervention

\begin{tabular}{|c|c|c|c|c|}
\hline \multirow[t]{2}{*}{ Variable } & \multicolumn{2}{|l|}{ Univariate } & \multicolumn{2}{|l|}{ Multivariate } \\
\hline & Unadjusted HR [95\% Cl] & p-value & Adjusted HR $[95 \% \mathrm{Cl}]$ & p-value \\
\hline \multicolumn{5}{|l|}{ Time-fixed variables } \\
\hline Sex, male & $0.98[0.88-1.08]$ & 0.646 & $1.06[0.95-1.19]$ & 0.292 \\
\hline Age, per 5 years & $1.07[1.05-1.10]$ & $<0.001$ & $1.07[1.04-1.10]$ & $<0.001$ \\
\hline Hypertension & $1.09[0.93-1.27]$ & 0.302 & $\mathrm{I} .02[0.87-1.20]$ & 0.082 \\
\hline Diabetes mellitus & $1.34[1.20-1.48]$ & $<0.001$ & $1.33[1.19-1.47]$ & $<0.001$ \\
\hline Atrial fibrillation & $1.27[1.08-1.50]$ & $<0.001$ & $1.16[0.98-1.37]$ & 0.090 \\
\hline Myocardial infarction & $1.42[1.28-1.57]$ & $<0.001$ & $1.42[1.29-1.58]$ & $<0.001$ \\
\hline \multicolumn{5}{|l|}{ Household Income } \\
\hline Low & I (Ref) & - & I (Ref) & - \\
\hline Middle & $0.99[0.87-1.12]$ & 0.863 & $1.03[0.91-1.17]$ & 0.637 \\
\hline High & $1.05[0.92-1.19]$ & 0.456 & $1.08[0.95-1.23]$ & 0.243 \\
\hline \multicolumn{5}{|l|}{ Time-dependent Variables } \\
\hline \multicolumn{5}{|l|}{ Antiplatelets } \\
\hline No aspirin, no ADPRB & I (Ref) & - & I (Ref) & - \\
\hline Aspirin only & $0.49[0.42-0.57]$ & $<0.001$ & $0.71[0.59-0.85]$ & $<0.001$ \\
\hline ADPRB only & $0.58[0.46-0.72]$ & $<0.001$ & $0.82[0.64-1.05]$ & 0.120 \\
\hline Aspirin plus ADPRB & $0.69[0.60-0.80]$ & $<0.001$ & $1.03[0.86-1.23]$ & 0.730 \\
\hline \multicolumn{5}{|l|}{ Statins } \\
\hline No statin & I (Ref) & - & I (Ref) & - \\
\hline Statin use & $0.69[0.62-0.78]$ & $<0.001$ & $0.72[0.63-0.8 I]$ & $<0.001$ \\
\hline Withdrawal & $1.90[1.56-2.32]$ & $<0.001$ & $1.87[1.52-2.29]$ & $<0.001$ \\
\hline
\end{tabular}

Note: Data are derived from multivariate time-dependent Cox proportional hazard regression model.

Abbreviations: ADPRB, adenosine diphosphate receptor blocker (clopidogrel, prasugrel, ticlopidine, ticagrelor); $\mathrm{Cl}$, confidence interval; $\mathrm{HR}$, hazard ratio. 
an inverse dose-response relationship between risk of the primary outcome and the intensity of statin. Adjusted HR [95\% CI] for withdrawal of "low-," "moderate-," and "highintensity" statin compared to that in the "no statin period" were 1.45 [0.74-2.86], 1.86 [1.49-2.32], and 2.61 [1.41-4.81], respectively (Figure 2). This finding suggested a greater rebound phenomenon with discontinuation of a highintensity statin compared to the rebound observed with discontinuation of a low-intensity statin. In the secondary outcome analysis for individual outcomes, statin withdrawal was significantly associated with increased risk for MI, coronary revascularization, and all-cause death (Table 3).

\section{Discussion}

The current study evaluated the beneficial effect and the withdrawal effect of statins in a long-term period after PCI based on real-world data. The current guidelines strongly recommend that high-intensity statin therapy should be continued before and after PCI to reduce the development of cardiovascular adverse events. ${ }^{25,26}$ Statins are generally well tolerated, but as many as $20-30 \%$ of patients report adverse events, and discontinuation of statin is very common in the clinical setting. ${ }^{6,12,27}$ About half of patients starting statin therapy discontinue the statin within the first year, and adherence to the statin decreases with time. ${ }^{28}$ Despite the emphasis on high-intensity statin use in the guidelines, adherence to high-intensity statins was relatively lower compared with adherence to low- to moderate-intensity statins, and there were more frequent adverse events. $^{23,29}$

Suboptimal use and discontinuation of statins have been important clinical issues. ${ }^{10,30,31}$ After myocardial infarction, early discontinuation of statins was a significant risk factor for worse clinical outcomes. ${ }^{32,33}$ In patients who had undergone major vascular surgery, statin discontinuation during the postoperative period was associated with a higher incidence of myocardial ischemia, nonfatal myocardial infarction, and cardiovascular death. ${ }^{9}$ Previous studies have focused mainly on the risk of statin discontinuation in the early phase after vascular events. Our longitudinal study evaluated the prognosis according to the use and discontinuation of statin during a long-term period. After PCI, patients who received statin treatment were at a significantly lower risk of the primary outcome, which means that statin therapy should be continued to maintain the protective effects on the cardiovascular system.

In this study, statin withdrawal led to an increased risk in excess of that of no statin use. More interestingly, the degree of withdrawal effect was correlated with the intensity of the statin last taken. These findings suggest the presence of a rebound phenomenon in the early period of statin withdrawal, known as "statin withdrawal syndrome." To maximize the cardiovascular benefits of statin therapy and to avoid the detrimental effect of statin withdrawal, there is

A

Risk period

no statin

statin

withdrawal

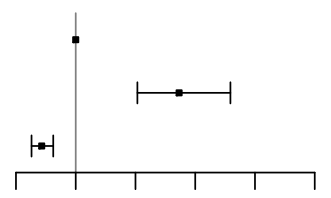

$\begin{array}{llllll}0.5 & 1 & 1.5 & 2 & 2.5 & 3\end{array}$

\section{B}

Risk period

no statin

statin

withdrawal, low-intensity withdrawal, moderate-intensity

withdrawal, high-intensity

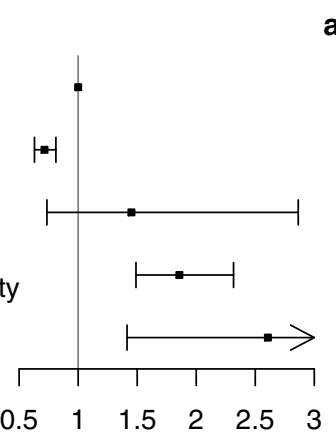

adjusted HR $\quad 95 \% \mathrm{Cl}$ p-value

1(Ref)

$0.72 \quad 0.63-0.81<0.001$

$1.87 \quad 1.52-2.29<0.001$

adjusted HR $\quad 95 \% \mathrm{Cl}$ p-value

1(Ref) - -

$0.71 \quad 0.63-0.81<0.001$

$1.45 \quad 0.74-2.86 \quad 0.283$

$1.86 \quad 1.49-2.32<0.001$

$2.61 \quad 1.41-4.81 \quad 0.002$

Figure 2 Risk for primary outcome according to statin treatment. (A) Adjusted HR for "statin use period" and "withdrawal period" compared to the "no statin period" (Ref). (B) Adjusted HR for statin withdrawal for "low-intensity", "moderate-intensity", and "high-intensity" statins compared to the "no statin period" (Ref). Data are derived from multivariate time-dependent Cox proportional hazard regression analysis adjusted for the variables listed in Table 2

Abbreviations: $\mathrm{Cl}$, confidence interval; $\mathrm{HR}$, hazard ratio. 
Table 3 Secondary Outcome Analysis for Individual Outcome

\begin{tabular}{|l|l|l|l|l|}
\hline Event of Interest & Myocardial Infarction & Coronary Revascularization & Stroke & All-Cause Death \\
\hline Risk period & & & & \\
No statin & I (Ref) & I (Ref) & I (Ref) & I (Ref) \\
Statin use & $0.84[0.64-1 . \mathrm{II}]$ & $0.79[0.66-0.94]$ & $0.73[0.33-1.6 \mathrm{I}]$ & $0.44[0.34-0.58]$ \\
Withdrawal & $2.1 \mathrm{I}[\mathrm{I} .39-3.20]$ & $2.43[1.8 \mathrm{I}-3.26]$ & $0.78[0.55-1.12]$ & $1.49[1.02-2.18]$ \\
\hline
\end{tabular}

Notes: Data are adjusted hazard ratio [95\% confidence interval] derived from multivariate time-dependent Cox proportional hazard regression models for individual outcomes. Adjustments were performed for same variables listed in Table 2.

a need to develop more practical interventions combining educational and behavioral components. ${ }^{28,34,35}$ The European Atherosclerosis Society Consensus Panel recommends brief discontinuation and re-initiation of statin therapy in patients with statin intolerance such as statin-attributed muscle symptoms. ${ }^{36}$ Based on the results of the present study, we found that a greater rebound effect occurs in association with withdrawal from high-intensity statins, and this is proportional to the statin intensity. Therefore, when statin intolerance develops (except in patients with serious side effects), it might be reasonable to down-titrate the dose initially rather than to abruptly stop the drugs or change to non-statins, especially in those taking high-intensity statins.

Some mechanisms have been proposed to explain the rebound phenomenon caused by statin withdrawal: 1) Induction of vascular dysfunction: The Rho family of guanosine triphosphates (GTP) is a family of small signaling $G$ proteins which modulate the levels of nitric oxide, production of reactive oxygen species, and levels of angiotensin II-AT1 receptors, endothelin-I, adhesion molecules, and inflammatory cytokines. ${ }^{12}$ Statins can block isoprenoid-dependent Rho membrane translocation and GTPbinding activity, which leads to upregulation of endothelial nitric oxide synthase expression and accumulation of nonisoprenylated Rho protein in the endothelial cytosol, inducing a vascular protective effect. ${ }^{37}$ Withdrawing statins can restore the availability of isoprenoids and result in a rebound activation of Rho and downregulation of endothelial nitric oxide production. Acute statin withdrawal increases angiotensin II receptor type 1 activity in smooth muscle cells and exacerbates vascular dysfunction. ${ }^{38} 2$ ) Rebound of inflammatory response: Statin withdrawal could induce a rebound phenomenon of the inflammatory response by increasing expression of inflammatory markers like C-reactive protein and interleukin-6. ${ }^{39}$ In an experimental study on human and rat vascular smooth muscle cells, statin withdrawal increased levels of proatherogenic substances, such as free radicals, monocyte chemoattractant protein 1 , and tissue factor gene expression. ${ }^{40}$ 3) Decreased angiogenesis: Statin discontinuation was purported to reduce angiogenesis, which could delay myocardial recovery after acute coronary syndrome. ${ }^{41}$

We should acknowledge the possible limitations of this study. This study had a retrospective cohort design; therefore, it might have confounding effects. Although there are previous validation studies for the outcome measures that used the NHIS database, non-hospitalized outcomes could not be captured with the limitations of the health claim database. We determined statin treatment by accessing the prescription data on the NHIS database. Actual statin use might have been different from the prescription data. Further large clinical and experimental studies are needed to evaluate the effect of "statin withdrawal" on cardiovascular prognosis and the underlying mechanisms.

\section{Conclusion}

After PCI, the use of statins showed a significant beneficial effect in the long-term period. Withdrawal of statins resulted in increased risk of cardiovascular events. Clinicians should take comprehensive measures to ensure that continued use of statins is maintained in all patients after PCI.

\section{Acknowledgments}

This study used the NHIS-NSC data (NHIS-2017-2-558) made by NHIS. This project was supported by grants of the Basic Science Research Program through the National Research Foundation of Korea (NRF) funded by the Ministry of Education (NRF-2017R1D1A1B03033382) and Ministry of Science and ICT (NRF2019R1F1A1062716). The funding bodies played no part in study design, data collection and analysis, decision to publish, or preparation of the manuscript.

\section{Disclosure}

The authors report no conflicts of interest in this work. 


\section{References}

1. Wang L, Peng P, Zhang O, et al. High-dose statin pretreatment decreases periprocedural myocardial infarction and cardiovascular events in patients undergoing elective percutaneous coronary intervention: a meta-analysis of twenty-four randomized controlled trials PLoS One. 2014;9(12):e113352. doi:10.1371/journal.pone.0113352

2. Feng WH, Chu CY, Hsu PC, et al. The effects of secondary prevention after coronary revascularization in Taiwan. PLoS One. 2019;14 (5):e0215811. doi:10.1371/journal.pone.0215811

3. Lloyd SM, Stott DJ, de Craen AJ, et al. Long-term effects of statin treatment in elderly people: extended follow-up of the PROspective Study of Pravastatin in the Elderly at Risk (PROSPER). PLoS One. 2013;8(9):e72642. doi:10.1371/journal.pone.0072642

4. Stone NJ, Robinson JG, Lichtenstein AH, et al. 2013 ACC/AHA guideline on the treatment of blood cholesterol to reduce atherosclerotic cardiovascular risk in adults: a report of the American College of Cardiology/American Heart Association Task Force on Practice Guidelines. J Am Coll Cardiol. 2014;63(25Pt B):2889-2934. doi:10.1016/j.jacc.2013.11.002

5. Lee SY, Oh SJ, Kim EJ, et al. Statin intensity and clinical outcome in patients with stable coronary artery disease and very low LDL-cholesterol. PLoS One. 2016;11(11):e0166246. doi:10.1371/ journal.pone. 0166246

6. Zhang H, Plutzky J, Skentzos S, et al. Discontinuation of statins in routine care settings: a cohort study. Ann Intern Med. 2013;158 (7):526-534. doi:10.7326/0003-4819-158-7-201304020-00004

7. Sawano M, Kohsaka S, Abe T, et al. Patterns of statin non-prescription in patients with established coronary artery disease: a report from a contemporary multicenter Japanese PCI registry. PLoS One. 2017;12(8):e0182687. doi:10.1371/journal.pone.0182687

8. Alwhaibi M, Altoaimi M, AlRuthia Y, et al. Adherence to statin therapy and attainment of LDL cholesterol goal among patients with type 2 diabetes and dyslipidemia. Patient Prefer Adherence. 2019;13:2111-2118. doi:10.2147/PPA.S231873

9. Le Manach Y, Godet G, Coriat P, et al. The impact of postoperative discontinuation or continuation of chronic statin therapy on cardiac outcome after major vascular surgery. Anesth Analg. 2007;104 (6):1326-1333, table of contents. doi:10.1213/01.ane.000026 3029.72643.10

10. Bansilal S, Castellano JM, Garrido E, et al. Assessing the impact of medication adherence on long-term cardiovascular outcomes. $J \mathrm{Am}$ Coll Cardiol. 2016;68(8):789-801. doi:10.1016/j.jacc.2016.06.005

11. Giral P, Neumann A, Weill A, Coste J. Cardiovascular effect of discontinuing statins for primary prevention at the age of 75 years: a nationwide population-based cohort study in France. Eur Heart $J$. 2019;40(43):3516-3525. doi:10.1093/eurheartj/ehz458

12. Pineda A, Cubeddu LX. Statin rebound or withdrawal syndrome: does it exist? Curr Atheroscler Rep. 2011;13(1):23-30. doi:10.1007/ s11883-010-0148-x

13. Heeschen C, Hamm CW, Laufs U, Bohm M, Snapinn S, White HD. Withdrawal of statins in patients with acute coronary syndromes. Circulation. 2003;107(3):e27. doi:10.1161/01.CIR.0000050552.32 300.93

14. Heeschen C, Hamm CW, Laufs U, et al. Withdrawal of statins increases event rates in patients with acute coronary syndromes. Circulation. 2002;105(12):1446-1452. doi:10.1161/01.CIR.000001 2530.68333.C8

15. Lee J, Lee JS, Park SH, Shin SA, Kim K. Cohort profile: the National Health Insurance Service-National Sample Cohort (NHIS-NSC), South Korea. Int J Epidemiol. 2016. doi:10.1093/ije/dyv319

16. Sung J, Hong KP. Descriptive Study on the Korean Status of Percutaneous Coronary Intervention Using National Health Insurance Service-National Sample Cohort (NHIS-NSC) database: focused on temporal trend. Korean Circ J. 2019;49(12):1155-1163. doi: $10.4070 / \mathrm{kcj} .2019 .0080$
17. Jee SH, Jang Y, Oh DJ, et al. A coronary heart disease prediction model: the Korean Heart Study. BMJ Open. 2014;4(5):e005025. doi:10.1136/bmjopen-2014-005025

18. Kimm H, Yun JE, Lee SH, Jang Y, Jee SH. Validity of the diagnosis of acute myocardial infarction in Korean National Medical Health Insurance Claims Data: the Korean Heart Study (1). Korean Circ J. 2012;42(1):10-15. doi:10.4070/kcj.2012.42.1.10

19. Park JK, Kim KS, Kim CB, et al. The accuracy of ICD codes for cerebrovascular diseases in medical insurance claims. Korean J Prev Med. 2000;33(1):76-82.

20. Park TH, Choi JC. Validation of stroke and thrombolytic therapy in Korean National Health Insurance Claim Data. J Clin Neurol. 2016;12(1):42-48. doi:10.3988/jen.2016.12.1.42

21. Rim TH, Han J, Choi YS, et al. Retinal artery occlusion and the risk of stroke development: twelve-year nationwide cohort study. Stroke. 2016;47(2):376-382. doi:10.1161/STROKEAHA.115.010828

22. Shin JY, Roughead EE, Park BJ, Pratt NL. Cardiovascular safety of methylphenidate among children and young people with attention-deficit/hyperactivity disorder (ADHD): nationwide self controlled case series study. BMJ. 2016;353:i2550. doi:10.1136/bmj. i 2550

23. Kim J, Lee HS, Nam CM, Heo JH. Effects of statin intensity and adherence on the long-term prognosis after acute ischemic stroke. Stroke. 2017;48(10):2723-2730. doi:10.1161/STROKEAHA.117.018140

24. Kang YM, Kim YJ, Park JY, Lee WJ, Jung CH. Mortality and causes of death in a national sample of type 2 diabetic patients in Korea from 2002 to 2013. Cardiovasc Diabetol. 2016;15(1):131. doi:10.1186/s12933-016-0451-0

25. Levine GN, Bates ER, Blankenship JC, et al. 2011 ACCF/AHA/ SCAI guideline for percutaneous coronary intervention. A report of the American College of Cardiology Foundation/American Heart Association Task Force on Practice Guidelines and the Society for Cardiovascular Angiography and Interventions. $J$ Am Coll Cardiol. 2011;58(24):e44-122. doi:10.1016/j.jacc.2011.08.007

26. Neumann FJ, Sousa-Uva M, Ahlsson A, et al. 2018 ESC/EACTS guidelines on myocardial revascularization. Eur Heart J. 2019;40 (2):87-165.

27. Katzmann JL, Mahfoud F, Bohm M, Schulz M, Laufs U. Association of medication adherence and depression with the control of low-density lipoprotein cholesterol and blood pressure in patients at high cardiovascular risk. Patient Prefer Adherence. 2019;13:9-19. doi:10.2147/PPA.S182765

28. Maningat $\mathrm{P}$, Gordon BR, Breslow JL. How do we improve patient compliance and adherence to long-term statin therapy? Curr Atheroscler Rep. 2013;15(1):291. doi:10.1007/s11883-012-0291-7

29. Virani SS, Woodard LD, Akeroyd JM, Ramsey DJ, Ballantyne CM, Petersen LA. Is high-intensity statin therapy associated with lower statin adherence compared with low- to moderate-intensity statin therapy? Implications of the 2013 American College of Cardiology/ American Heart Association Cholesterol Management Guidelines. Clin Cardiol. 2014;37(11):653-659. doi:10.1002/clc.22343

30. Degli Esposti L, Saragoni S, Batacchi P, et al. Adherence to statin treatment and health outcomes in an Italian cohort of newly treated patients: results from an administrative database analysis. Clin Ther. 2012;34(1):190-199. doi:10.1016/j.clinthera.2011.12.011

31. Tziomalos K, Athyros VG, Mikhailidis DP. Statin discontinuation: an underestimated risk? Curr Med Res Opin. 2008;24(11):3059-3062. doi:10.1185/03007990802469102

32. Daskalopoulou SS, Delaney JA, Filion KB, Brophy JM, Mayo NE, Suissa S. Discontinuation of statin therapy following an acute myocardial infarction: a population-based study. Eur Heart J. 2008;29 (17):2083-2091. doi:10.1093/eurheartj/ehn346

33. Spencer FA, Fonarow GC, Frederick PD, et al. Early withdrawal of statin therapy in patients with non-ST-segment elevation myocardial infarction: national registry of myocardial infarction. Arch Intern Med. 2004;164(19):2162-2168. doi:10.1001/archinte.164.19.2162 
34. Mann A, Esse TW, Serna O, Castel LD, Abughosh SM. Effectiveness of mailed letters to improve medication adherence among medicare advantage plan participants with chronic conditions. Patient Prefer Adherence. 2019;13:37-46. doi:10.2147/PPA.S185848

35. Armstrong SO, Little RA. Cost effectiveness of interventions to improve adherence to statin therapy in ASCVD patients in the United States. Patient Prefer Adherence. 2019;13:1375-1389. doi:10.2147/PPA.S213258

36. Stroes ES, Thompson PD, Corsini A, et al. Statin-associated muscle symptoms: impact on statin therapy-European Atherosclerosis Society Consensus Panel Statement on Assessment, Aetiology and Management. Eur Heart J. 2015;36(17):1012-1022. doi:10.1093/eurheartj/ehv043

37. Laufs U, Endres M, Custodis F, et al. Suppression of endothelial nitric oxide production after withdrawal of statin treatment is mediated by negative feedback regulation of rho GTPase gene transcription. Circulation. 2000;102(25):3104-3110. doi:10.1161/01. CIR.102.25.3104
38. Touyz RM, Schiffrin EL. Signal transduction mechanisms mediating the physiological and pathophysiological actions of angiotensin II in vascular smooth muscle cells. Pharmacol Rev. 2000;52(4):639-672.

39. Li JJ, Li YS, Chu JM, et al. Changes of plasma inflammatory markers after withdrawal of statin therapy in patients with hyperlipidemia. Clin Chim Acta. 2006;366(1-2):269-273. doi:10.1016/j.cca.2005. 10.021

40. Brandes RP, Beer S, Ha T, Busse R. Withdrawal of cerivastatin induces monocyte chemoattractant protein 1 and tissue factor expression in cultured vascular smooth muscle cells. Arterioscler Thromb Vasc Biol. 2003;23(10):1794-1800. doi:10.1161/01.ATV.00000921 26.25380.BC

41. Chen J, Zhang ZG, Li Y, et al. Statins induce angiogenesis, neurogenesis, and synaptogenesis after stroke. Ann Neurol. 2003;53 (6):743-751. doi:10.1002/(ISSN)1531-8249

\section{Publish your work in this journal}

Patient Preference and Adherence is an international, peer-reviewed, open access journal that focuses on the growing importance of patient preference and adherence throughout the therapeutic continuum. Patient satisfaction, acceptability, quality of life, compliance, persistence and their role in developing new therapeutic modalities and compounds to optimize clinical outcomes for existing disease states are major areas of interest for the journal. This journal has been accepted for indexing on PubMed Central. The manuscript management system is completely online and includes a very quick and fair peer-review system, which is all easy to use. Visit http:// www.dovepress.com/testimonials.php to read real quotes from published authors. 